\title{
On the spider fauna (Arachnida: Aranei) of the Mordovian State Reserve, Russia: preliminary results
}

\author{
О фауне пауков (Arachnida: Aranei) Мордовского \\ государственного заповедника: предварительные результаты
}

\author{
K.G. Mikhailov ${ }^{1}$, E.E. Trushina \\ К.Г. Михаймов ${ }^{1}$, Е.Э. Трушина
}

\author{
Zoological Museum, Moscow Lomonosov State University, Bolshaya Nikitskaya Str.6, Moscow 125009 Russia. \\ 'E-mail: mikhailov2000@gmail.com \\ Зоологический музей МГУ им. М.В. Ломоносова, ул. Большая Никитская, 6, Мрсква 125009 Россия. \\ ${ }^{1}$ E-mail: mikhailov2000@gmail.com
}

KEY WORDS: biodiversity, spiders, fauna, Mordovian State Reserve, Russian Plain.

КЛЮЧЕВЫЕ СЛОВА: биоразнообразие, пауки, фауна, Мордовский государственный заповедник, Русская равнина.

ABSTRACT. A preliminary list of spiders of the Mordovian State Reserve (Russian Plain, the southern part of forest zone) is presented and includes 142 species of 17 families, which seems to be a half of the expected spider diversity of the reserve. The gained results are briefly discussed and a complete list of the currently known spider species of Mordovian Republic (in total, 161 species) is provided.

РЕЗЮМЕ. Предварительный список пауков Мордовского государственного заповедника (Русская равнина, южная часть лесной зоны) включает 142 вида из 17 семейств, что, по-видимому, составляет половину ожидаемого разнообразия пауков заповедника. Полученные результаты кратко обсуждаются; приведен полный также список пауков Республики Мордовия, составляющий 161 вид.

The Mordovian State Reserve (MSR) is situated in the southern part of the forest zone of Russian Plain, although Mordovian Republic lies primarily in the forest-steppe zone. Biodiversity studies of the MSR were launched since its establishment in 1936, with first results being summarized already in two years [Fauna Mordovskogo..., 1938]. A special paper devoted to insect fauna also included several species of mites, harvestmen, pseudoscorpions and millipedes [Redikortsev, 1938], but no spiders. To date no special paper on the spider fauna of the MSR has been published. In two local papers, 40 spider species of 15 families were reported from Mordovian Republic, mostly from wheat fields and without proper locality information [Timraleev, 1998; Budilov, Timoshkina, 2010]. These species are incorporated in the spider list given below.

The aim of this paper is to provide a preliminary list of MSR spiders with detailes of their habitat preferences according to the ten plant communities studied, against the background of the Mordovian Republic spider fauna.

\section{Material and Methods}

The field collecting was conducted as a student work of the second author (ET) in 2010 and 2011. Spiders were collected in the vicinity of Pushta settlement, Temnikov District, Mordovian Republic, $54.712524^{\circ} \mathrm{N}, 43.225708^{\circ} \mathrm{E}$. The following plant communities were surveyed (Figs 1-10): [1] hygrophytic meadow with scarce young pines; [2] mesophytic meadow with scarce young pines; [3] pine forest with scarce maple and rowen tree undergrowth; [4] mixed spruce forest with pines and singular maples; [5] mixed hygrophytic Alnus forest with birches and pines; [6] Alnus forest; [7] mixed birch forest with pines; [8] broadleaved forest with maples, oaks, birches and lime trees; [9] birch and lime tree plantation within the settlement; [10] mesophytic meadow with Artemisia. The respective numbers given in square brackets are indicated on the locality labels retained in studied sample tubes, as well as they are given in the following species (see below). Since the second part of July 2010 was extremely dry followed by fires in the most part of European Russian forest zone, spider collecting was ceased at the beginning of August. One of the studied plant communities [10] was destroyed by fire but recovered in 2011 (see the respective photo on Fig. 10). The number of specimens collected in 2010 is considerably lower than that of 2011.

The following spider list includes all the currently available data, both original (from the MSR) and literature-derived, on the spiders of Mordovian Republic (MR). Unfortunately, the literature-derived data remain questionnable, because (1) they originated from not peer-reviewed sources, (2) none of the professional 


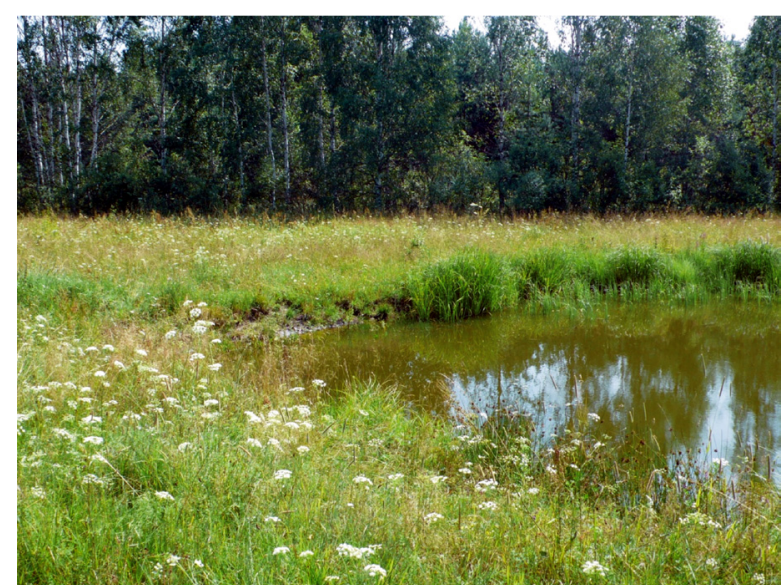

1
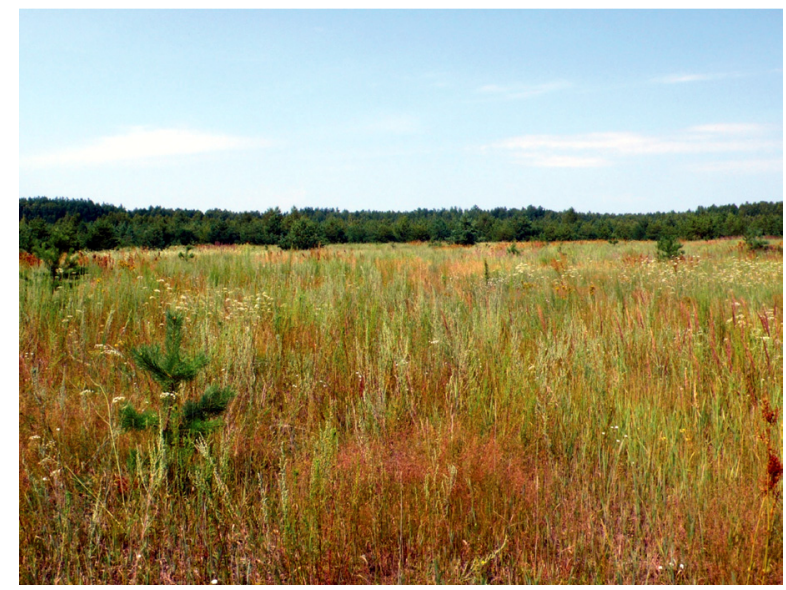

2

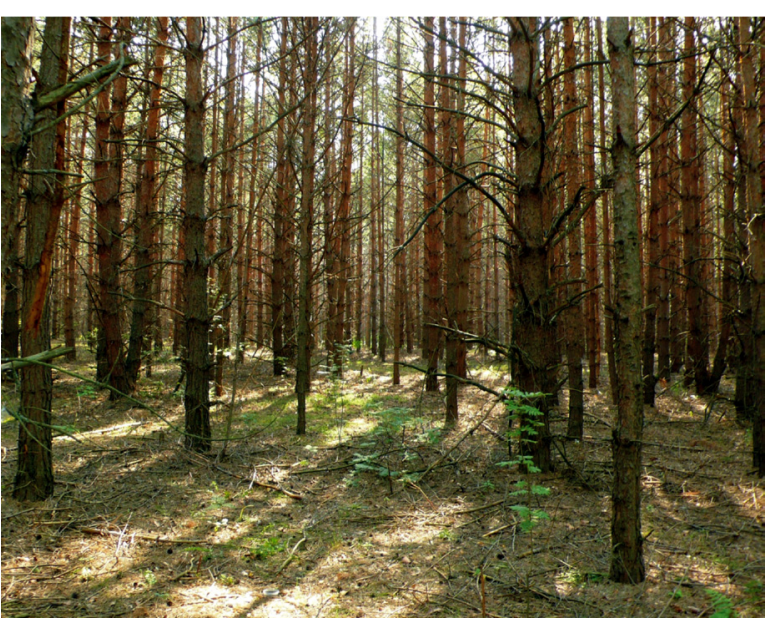

3

Figs 1-10. Plant communities of Mordovian State Reserve studied: 1 - hygrophytic; 2 - mesophytic meadow; 3 - pine forest: 4 - mixed spruce forest; 5 - mixed hygrophytic Alnus forest; 6 - Alnus forest; 7 - mixed birch forest; 8 - broadleaved forest; 9 - birch and lime tree plantation; $10-$ mesophytic meadow with Artemisia.

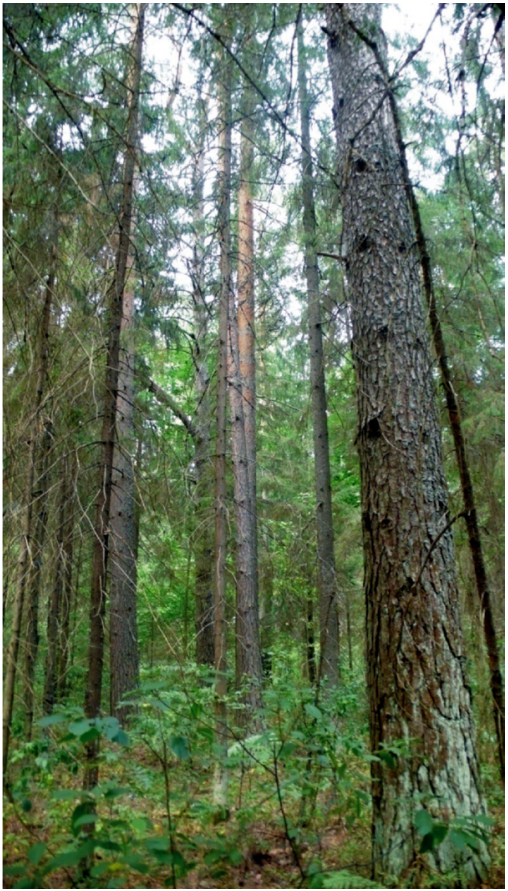

4

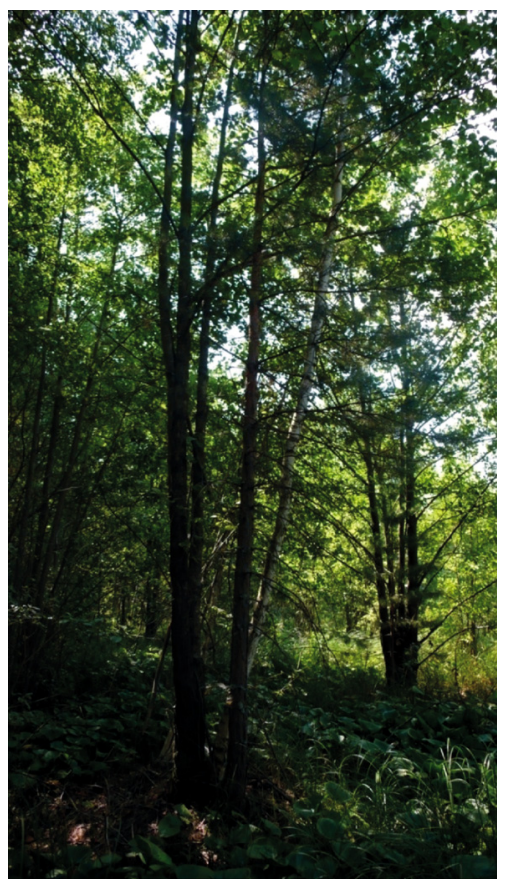

5

Рис. 1-10. Исследованные растительные сообщества Мордовского государственного заповедника: 1 - гигрофитный луг; 2 - мезофитный луг; 3 - сосновый лес; 4 - смешанный еловый лес; 5 - смешанный гигрофитный ольховый лес: 6 ольховый лес: 7 - смешанный березовый лес; 8 - широколиственный лес; 9 - насаждения березы и липы; 10 - мезофитный луг с полынью. 


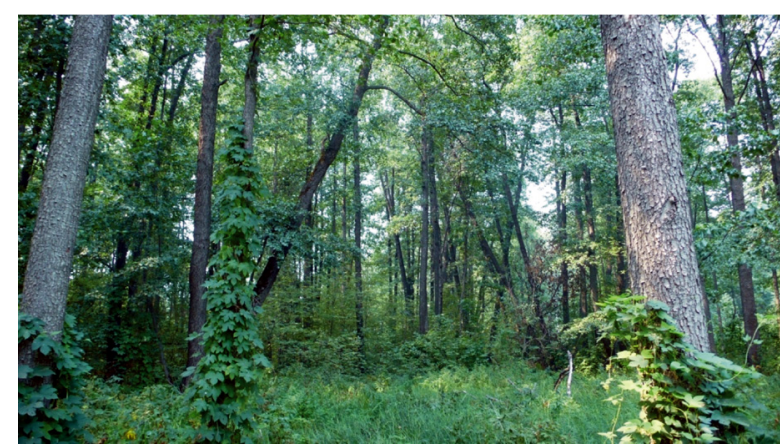

6

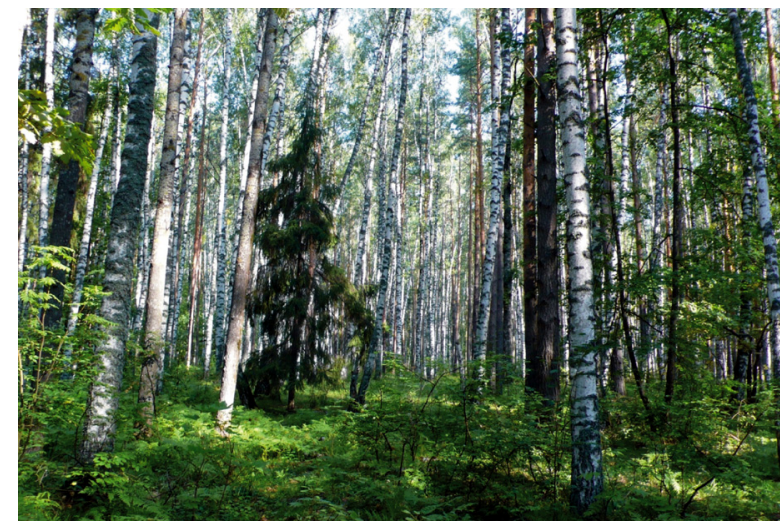

7

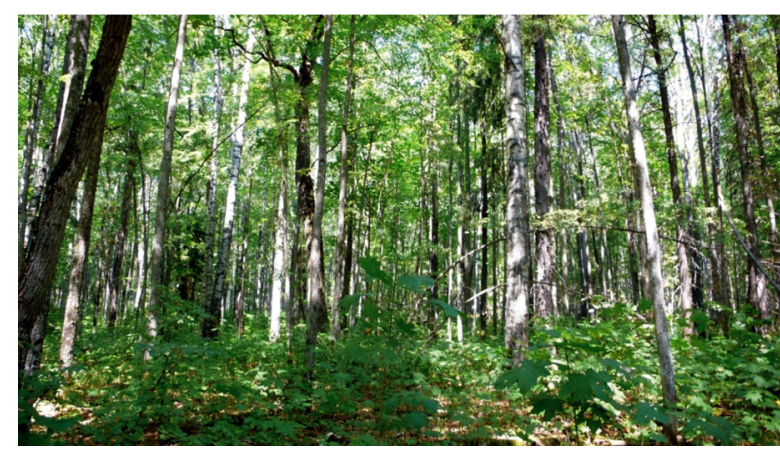

8

arachnologists was involved in indentification, and (3) no reference material is available. The majority of MSR spiders have been identified by the first author (KM), several linyphiids have been determined by $\mathrm{Dr}$ A.V. Tanasevitch (Moscow, Russia). In the following list, spider families are listed alphabetically, the number of MSR species is given in brackets following the respective family name. The species recorded from MR for the first time are marked with asterisks $(*)$; the species that have been reported on the basis of literature data [Timraleev, 1998; Budilov, Timoshkina, 2010] but absent from our list of the MSR fauna are marked with (-) and not counted in further discussion. Two species, Ballus depressus (Walckenaer, 1802) and Coriarachne depressa (C.L. Koch, 1837) have been added from the initial MSR spider collection kept in the Zoological Museum of the Moscow State University (ZMUM), identified by A. Chernov under the supervision of K.G.
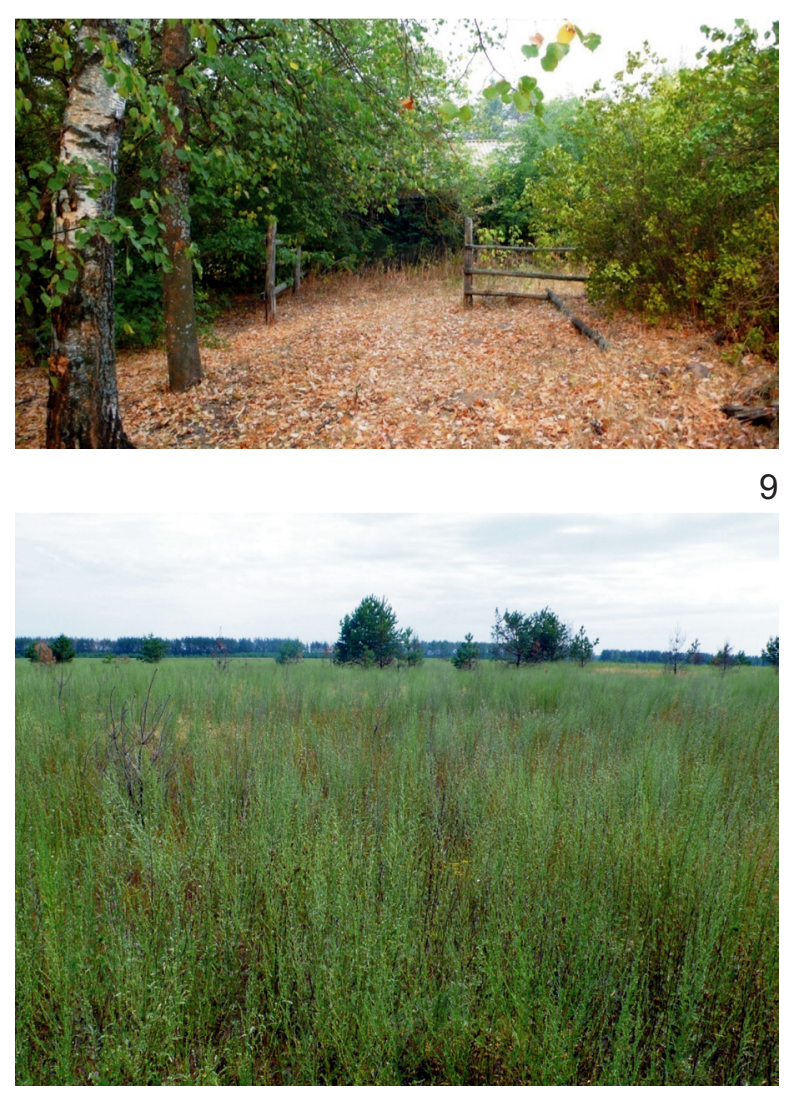

10

Figs 1-10 (continued).

Рис. 1-10 (продолжение).

Mikhailov in mid-1980ies. The following abbreviations are used in the species list: bc. - biocenometer, h.c. hand collecting, p.t. - pitfall traps, sw. - sweeping. The material studied is kept in the ZMUM, and a small reference collection will be handed over to the MSR.

\section{List of spiders}

\section{AGELENIDAE (1)}

(-) Agelena labirynthica (Clerck, 1758). MR: wheat fields [Timraleev, 1998].

Tegenaria domestica (Clerck, 1758)* - $1 \sigma^{\top}$ [9], p.t., 7-17.07.2010.

ARANEIDAE (8)

Araneus diadematus Clerck, $1758-19$ [4], p.t., 17-27.07.2010; 1 ○ sbad. [4], h.c., 17-27.07.2010; $1 \sigma^{7}$ [4], h.c., 10.06-31.08.2011; $1 \sigma^{7}$ [7], sw., 21.08. 
2011; 1 \% , 1 juv. [9], h.c., 10.06-31.08.2011. MR: Ardatovo Distr.: Smol'kovo Vil. [Budilov, Timoshkina, 2010].

(-) Argiope bruennichi (Scopoli, 1772). MR: wheat fields [Timraleev, 1998].

Cercidia prominens (Westring, 1851)* - $1 \bigcirc^{7}[3]$, p.t., 11-21.06.2011; 1 O $^{7}$ sbad. [5], sw., 12.07.2011. Cyclosa conica (Pallas, 1772)* -1 + [4], sw., 12.06.2011.

Hypsosinga pygmaea (Sundevall, 1831) - 1 \& [8], sw., 22.06.2011. MR: wheat fields [Timraleev, 1998].

Larinioides cornutus (Clerck, 1758) - 1 ㅇ [1], sw., 22.06.2011; 1 O $^{7}$ [1], sw., 12.07.2011. MR: wheat fields [Timraleev, 1998].

(-) Larinioides ixobolus (Thorell, 1873). MR: wheat fields [Timraleev, 1998].

Larinioides patagiatus (Clerck, 1758)* -1 [6], sw., 7-17.07.2010.

Mangora acalypha (Walckenaer, 1802) - 1 [1], sw., 12.06.2011; 2 of [1], sw., 22.06.2011; 1 juv. [3], sw., 1.08.2011; [3], sw., 11.08.2011; 1 ㅇ [9], sw., 12.06.2011; 1 + [9], sw., 2.07.2011; 1 juv. [9], sw., 1.08.2011. MR: wheat fields [Timraleev, 1998].

(-) Neoscona adianta (Walckenaer, 1802). MR: wheat fields [Timraleev, 1998].

Singa nitidula C.L. Koch, $1844^{*}-1$ \& [9], h.c., 10.06-31.08.2011.

\section{CLUBIONIDAE (4)}

Cheiracanthium virescens (Sundevall, 1832)* $1 \sigma^{\top}$ [2], p.t., 1-11.07.2011.

Clubiona caerulescens L. Koch, 1867* - $10^{7}[3]$, p.t., 11-21.06.2011; 1 ㅇ [7], bc., 7.08.2011.

Clubiona diversa O. Pickard-Cambridge, 1862* 3 우 [2], p.t., 7-17.07.2010; 2 우 [2], p.t., 1727.07.2010; 2 +o [2], p.t., 17-27.07.2010.

Clubiona lutescens Westring, 1851* -1 \% [5], sw., 12.07.2011; 1 \% [6], bc., 7.07.2011; 1 ○ $^{7}$ [9], h.c., 10.06-31.08.2011.

Clubiona pallidula $($ Clerck, 1758)* — 1 \& [9], p.t., 17-27.07.2010.

(-) Clubiona stagnatilis Kulczyński in Chyzer et Kulczyński, 1897. MR: Ardatovo Distr.: Smol'kovo Vil. [Budilov, Timoshkina, 2010].

CORINNIDAE (1)

Phrurolithus festivus (C.L. Koch, 1835)* -1 ㅇ [7], bc., 28.06.2011.

DICTYNIDAE (3)

(-) Dictyna arundinacea (1., 1758). MR: wheat fields [Timraleev, 1998].

Dictyna pusilla Thorell, 1856* -1 † [9], sw., 8.07.2011.

Dictyna uncinata Thorell, 1856* - $1{ }^{7}$ [4], sw., 12.06.2011; 1 ○ [4], sw., 2.07.2011.

Lathys nielseni (Schenkel, 1932)* -1 q [3], bc., 14.06.2011; 1 + [3], bc., 14.07.2011.

GNAPHOSIDAE (14)

Drassodes pubescens (Thorell, 1856)* $-2 \sigma^{7} \sigma^{7}$ [1], p.t., 11-21.06.2011; 1 ठ $^{7}[1]$, p.t., 21.06-1.07.2011; $1 \sigma^{7}[1]$, p.t., 1-11.07.2011; 1 ㅇ [2], p.t., 17-27.07.2010.
Drassodes villosus (Thorell, 1856)* — 1 ㅇ [9], h.c., summer 2011.

Drassyllus praeficus (L. Koch, 1866) - $1 \sigma^{7}$ [9], p.t., 21.06-1.07.2011. MR: wheat fields [Timraleev, 1998].

Drassyllus pusillus (C.L. Koch, 1833)* - $10^{7}[1]$, p.t., 11-21.06.2011; 1 [ [1], p.t., 11-21.06.2011; 2 O $^{7}$ [1], p.t., 21.06-1.07.2011; 1 O $^{T}$ [5], p.t., 11-21.06.2011; $2 \sigma^{7} \sigma^{7}[6]$, p.t., 11-21.06.2011; $1 \sigma^{7}$ [9], p.t., 21.061.07.2011; 1 + [9], p.t., 1-11.07.2011.

Gnaphosa bicolor (Hahn, 1833)* - $1 \sigma^{7}$ [2], p.t., 11-21.06.2011; 1 ○ [2], p.t., 21.06-1.07.2011; 1 ऊ [5], p.t., 11-21.06.2011; $1 \sigma^{7}$ [9], p.t., 11-21.06.2011.

(-) Gnaphosa lucifuga (Walckenaer, 1802). MR: wheat fields [Timraleev, 1998].

Haplodrassus cognatus (Westring, 1861)* - 1 q [4], p.t., 17-27.07.2010; 1 क [7], p.t., 7-17.07.2010; 1 [ [7], p.t., 11-21.06.2011.

Haplodrassus signifer (C.L. Koch, 1839) - $2 \sigma^{7} \sigma^{7}$, 1 ○ [2], p.t., 11-21.06.2011; 1 [2], p.t., 21.06$1.07 .2011 ; 1 \sigma^{7}$ [2], p.t., 1-11.07.2011; $1 \sigma^{7}$ [5], p.t., 21.06-1.07.2011; $1 \Im^{7}, 6$ oq [8], p.t., 11-21.06.2011; 1 ㅇ [9], p.t., 21.06-1.07.2011. MR: wheat fields [Timraleev, 1998].

Haplodrassus silvestris (Blackwall, 1833)* - $3 \sigma^{7} \sigma^{7}$ [3], p.t., 11-21.06.2011; 3 О $^{\top}$ [4], p.t., 11-21.06.2011; 1 $\sigma^{7}$ [5], p.t., 11-21.06.2011; 1 o, 1 juv. [7], p.t., 717.07.2010; 1 ㅇ [9], p.t., 7-17.07.2010.

Haplodrassus soerenseni (Strand, 1900)* - $2 \sigma^{7} \sigma^{7}$

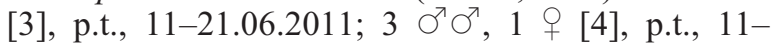
21.06.2011.

Micaria formicaria (Sundevall, 1831)* - $1 O^{7}[1]$, p.t., 1-11.07.2011; 1 9 [9], p.t., 1-11.07.2011.

Micaria pulicaria (Sundevall, 1831)* - 1 \% [7], bc., 7-17.07.2010; 1 O', 1 \% [7], bc., 28.07.2011.

(-) Micaria rossica Thorell, 1875. MR: wheat fields [Timraleev, 1998].

Zelotes apricorum (L. Koch, 1876) - $1 \bigcirc^{7}$ [9], p.t.,

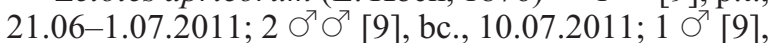
bc., 9.08.2011. MR: wheat fields [Timraleev, 1998].

Zelotes longipes (L. Koch, 1866)* - 2 O $^{\top} \sigma^{7}[2]$, p.t., 7-17.07.2010; 1 \% [2], p.t., 17-27.07.2010; 1 \% [2], p.t., 11-21.06.2011; 3 ठ7 ठ [10], p.t., 7-17.07.2010. Zelotes subterraneus (C.L. Koch, 1833)* - $1 \sigma^{7}$ [3], p.t., 7-17.07.2010; 1 \% [7], 11-21.06.2011; 1 ○ [9], bc., 9.08.2011.

HAHNIIDAE (4)

Antistea elegans (Blackwall, 1841)* -1 [1],

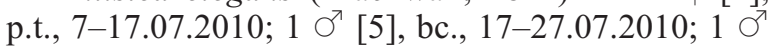
[8], bc., 27.07-6.08.2010.

Hahnia nava (Blackwall, 1841)* -1 + [2], bc., 17-27.07.2010.

Hahnia ononidum Simon, $1875^{*}-1$ + $[1]$, bc., 27.07-6.08.2010.

Hahnia pusilla C.L. Koch, $1841^{*}-1$ 잉, bc., 3.07.2011.

\section{LINYPHIIDAE (37)}

Abacoproeces saltuum (L. Koch, 1872)* - $1 \sigma^{7}$ [3], p.t., 11-21.06.2011; 1 + [3], p.t., 11-21.06.2011; $1 \bigcirc^{7}$ [3], p.t., 21.06-1.07.2011; 1 ㅇ [3], p.t., 21.06- 


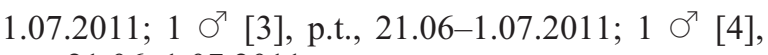
p.t., 21.06-1.07.2011. Agyneta affinis (Kulczyński, 1898)* - 1 [9], bc.,

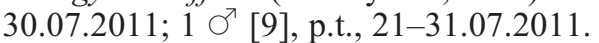
Agyneta cf. affinis - 1 [9], sw., 2.07.2011; 1 ㅇ

[9], bc., 20.07.2011. (-) Agyneta rurestris (C.L. Koch, 1836). MR: wheat fields [Timraleev, 1998]. Agyneta sp. (different species) -2 +o [6], bc., 17.06.2011. Allomengea vidua (L. Koch, 1879)* $-1+$ [5], bc., 7-17.07.2010; 3 Oᄋ [5], p.t., 7-17.07.2010; 4 Oๆ [5], p.t., 17-27.07.2010. Bolyphantes alticeps (Sundevall, 1832)* -1 ㅇ

[7], sw., 7-17.07.2010; 1 + [8], sw., 7-17.07.2010. Bolyphantes luteolus (Blackwall, 1833)* - 1 q

[7], p.t., 17-27.07.2010. Centromerus brevivulvatus F. Dahl, 1912* - 19

[3], bc., 14.07.2011. Centromerus sylvaticus (Blackwall, 1841)* - 19

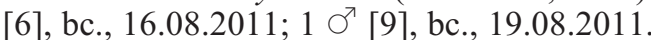
Cnephalocotes obscurus (Blackwall, 1834)* - 1 o [9], bc., 29.08.2011. Diplocentria bidentata (Emerton, 1882)* -1 q

[3], bc., 14.07.2011. Diplocephalus dentatus Tullgren, $1955^{*}-2$ 우

[5], bc., 16.06.2011; 1 + [5], bc., 16.07.2011. Diplocephalus picinus (Blackwall, 1841)* - 1 우

[6], bc., 7.07.2011. Diplostyla concolor (Wider, 1834)* — $1+[6]$, bc. 7.07.2011.

Floronia bucculenta (Clerck, 1758)* — 1 ○ [6], sw., 27.07-6.08.2010. Gonatium rubellum (Blackwall, 1841)* - 19 [6], bc., 7-17.07.2010; 1 + [6], bc., 17-27.07.2010; 1 \% [6], p.t., 1-11.07.2011. Gongylidiellum latebricola (O. Pickard-Cambridge, 1871)* - 1 \% [4], bc., 24.08.2011.

Gongylidiellum murcidum Simon, 1884* -2 우

[1], bc., 7-17.07.2010; 1 [ [5], bc., 7-17.07.2010; 1 \% [5], bc., 6.07.2011; 1 [6], bc., 7-17.07.2010; 1 \% [6], bc., 16.08.2011.

Gongylidium rufipes (L., 1758)* - 1 ○ [6], sw.,

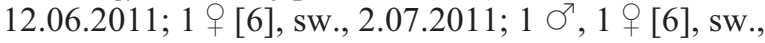
21.08.2011.

Helophora insignis (Blackwall, 1841)* - 1 juv. [4], sw., 27.07-6.08.2010; 1 † sbad. [6], sw., 1727.07.2010; 1 O $^{\top}$ [6], sw., 11.08.2011; 1 o sbad, 1 ㅇ sbad. [8], sw., 27.07-6.08.2010.

Leptorhoptrum robustum (Westring, 1851)* - 1

$\sigma^{7}$ [6], p.t., $11-21.06 .2011 ; 1$ 王 [6], p.t., 21.061.07.2011.

Linyphia hortensis Sundevall, 1830* - $10^{7}$ [4], p.t., 11-21.06.2011.

Linyphia triangularis (Clerck, 1758)* - 1 ○ [3], sw., 7-17.07.2010; 1 + [3], sw., 17-27.07.2010; 2 \% [3], sw., 27.07-6.08.2010; 1 juv. [3], sw., 22.06.2011; 1 juv. [3], sw., 2.07.2011; 1 + [3], sw., 12.07.2011; 3 of

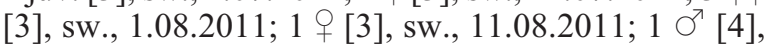
sw., 7-17.07.2010; 1 +, $1 \bigcirc^{\text {T }}$ sbad, 1 juv. [4], h.c., 1727.07.2010; 1 + [4], sw., 27.07-6.08.2010; 1 + [4],
SW., 21.08.2011; 4 우 [5], sw., 17-27.07.2010; 1 ○ $^{7}$ [5], sw., 22.07.2011; 1 q, 2 qo sbad. [5], sw., 22.07.2011; 1 क [5], sw., 11.08.2011; 3 क० [6], sw.,

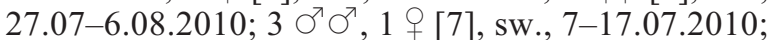
2 우 [7], bc., 17-27.07.2010; 1 juv. [7], h.c., 1727.07.2010; 2 ○० [7], sw., 27.07-6.08.2010; 4 juv. [7], sw., 12.07.2011; $1 \bigcirc^{7}, 7$ juv. [7], sw., 22.07.2011; 2 O$^{7} \sigma^{7}$, 3 우 [7], sw., 1.08.2011; 2 ठ $\sigma^{7}$ [7], sw., 11.08.2011; 3 우 [7], sw., 11.08.2011; 2 우 [7], sw., 21.08.2011; $1 \mathrm{O}^{7}$ sbad. [9], sw., 7-17.07.2010.

Macrargus rufus (Wider, 1834)* - 1 \% [4], bc., 24.08.2011.

Megalepthyphantes pseudocollinus Saaristo, $1997 *-1$ \% [9], p.t., 7-17.07.2010.

Micrargus herbigradus (Blackwall, 1854)* - 2 90

[1], bc., 7-17.07.2010; 3 ㅇ [1], bc., 17-27.07.2010; 1 o [6], bc., 7-17.07.2010.

Minyriolus pusillus (Wider, 1834)* $-1+[3]$, bc., 7-17.07.2010; 2 우 [3], bc., 7-17.07.2010; 1 ○, 3 우 [3], bc., 14.06.2011; 1 [ [3], bc., 14.06.2011; 1 \% [3], bc., 24.06.2011; $1 \bigcirc^{7}$ [3], bc., 24.07.2011; 2 우 [3], bc., 13.08.2011; 1 + [7], bc., 7-17.07.2010.

Neriene clathrata (Sundevall, 1830)* - 1 + [9], p.t., 7-17.07.2010.

Neriene emphana (Walckenaer, 1841)* — 1 \% [4], sw., 7-17.07.2010.

(-) Oedothorax apicatus (Blackwall, 1850). MR: wheat fields [Timraleev, 1998].

Panamomops mengei Simon, 1926* - $1+$ [4], bc., 7-17.07.2010; 1 ㅇ [4], bc., 5.07.2011.

Tapinocyba biscissa (O. Pickard-Cambridge, 1872)* -1 O [6], bc., 17.06.2011.

Tapinocyba insecta (L. Koch, 1869)* - 1 \% [4], bc., 15.06.2011; 1 + [4], bc., 5.07.2011.

Tapinocyba pallens (O. Pickard-Cambridge, 1872)*

-1 [4], bc., 25.07.2011; 1 \% [9], bc., 19.08. 2011.

Tiso vagans (Blackwall, 1834)* -1 \% [9], bc., 30.07.2011; 1 + [9], bc., 29.08.2011.

Troxochrus scabriculus (Westring, 1851)* -2 우 [9], bc., 30.07.2011; 1 ๙ $^{7}, 1$ 9 [9], bc., 19.08.2011; 1 O (f. cirrifrons) [9], bc., 19.08.2011; 1 ๆ [9], bc., 19.08.2011; 1 ○ [9], bc., 29.08.2011; 2 +o [9], bc., 29.08.2011.

Walckenaeria antica (Wider, 1834)* $-3 \bigcirc^{7} \bigcirc^{7}, 1$ 우

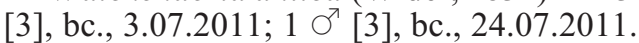

Walckenaeria atrotibialis O. Pickard-Cambridge, 1878* - 1 \% [4], bc., 15.06.2011.

Walckenaeria cucullata (C.L. Koch, 1836)* - 1 ㅇ [4], p.t., 21.06-1.07.2011.

Walckenaeria furcillata (Menge, 1869)* - 1 o [6], bc., 6.08.2011.

\section{LIOCRANIDAE (1)}

Agroeca brunnea (Blackwall, 1833)* - 1 \% [1], p.t., 21.06-1.07.2011; 2 O+ [3], p.t., 7-17.07.2010; 1 \% [3], p.t., 11-21.06.2011; 2 o+ [3], p.t., 21.06-1.07.2011; 1 [4], p.t., 11-21.06.2011; 1 \% [4], p.t., 21.061.07.2011; 3 +o [4], p.t., 1-11.07.2011; 2 +o [7], p.t., 21.06-1.07.2011.

\section{LYCOSIDAE (19)}

(-) Allohogna singoriensis (Laxmann, 1770). MR: wheat fields [Timraleev, 1998]. 
Arctosa leopardus (Sundevall, 1832)* - $1 \sigma^{7}[1]$, p.t., 21.06-1.07.2011.

Arctosa stigmosa (Thorell, 1875)* -1 + [1], p.t., 11-21.06.2011; 1 + [1], p.t., 21.06-1.07.2011.

Pardosa agrestis (Westring, 1861) - $1 \bigcirc^{7}, 1$ [8], p.t., 21.06-1.07.2011. MR: wheat fields [Timraleev, 1998, as both $P$. agrestis and P. monticola].

Pardosa fulvipes (Collett, 1875)* -1 i [1], p.t., 7-17.07.2010; 1 [1], sw., 17-27.07.2010; 3 ○ $^{7}, 1$ \% [1], p.t., 21.06-1.07.2011; 1 क [1], sw., 22.06.2011; 2 우 [1], sw., 2.07.2011; 2 우 [1], sw., 12.07.2011; 1 O [5], p.t., 11-21.06.2011; 1 + [9], h.c., 17-27.07.2010.

Pardosa lugubris (Walckenaer, 1802)* -1 + [2], p.t., 21-31.07.2011; 12 O $^{\top}$ [3], p.t., 11-21.06.2011;

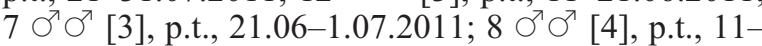

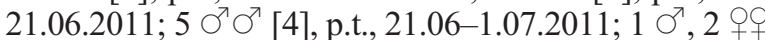
[5], p.t., 7-17.07.2010; 5 O+ [5], p.t., 17-27.07.2010;

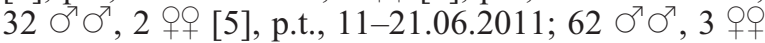
[5], p.t., 21.06-1.07.2011; $11 \sigma^{\top} \sigma^{\top}, 2$ +o [5], p.t., 111.07.2011; $1 \sigma^{7}$ [6], p.t., 21.06-1.07.2011; 2 우 [7], p.t., 7-17.07.2010; $27 \bigcirc^{7}, 3$ o 37$]$, p.t., 1121.06.2011; 17 O $^{7}, 3$ 우 [7], p.t., 21.06-1.07.2011; 2 우 [7], p.t., 1-11.07.2011; 9 우 [9], p.t., 717.07.2010; 2 우 [9], p.t., 17-27.07.2010; 1 \% 1 ㅇ [9], p.t., 11-21.06.2011; $17 \sigma^{7} \sigma^{7}$ [9], p.t., 21.061.07.2011; 2 o $\sigma^{7}$ [9], p.t., 1-11.07.2011; 1 \% [9], h.c., 10.06-31.08.2011.

Pardosa palustris (L., 1758)* -1 \% [1], p.t., $11-$ 21.06.2011; 2 우 [2], p.t., 7-17.07.2010; $6 \bigcirc^{7} \sigma^{7}, 3$ 우 [2], p.t., 11-21.06.2011; 1 q [2], p.t., 21.06-1.07.2011; 1 + [2], p.t., 1-11.07.2011; 6 О $^{7}, 6$ \% 6 [8], p.t., $11-$ 21.06.2011; 1 \% [8], p.t., 21.06-1.07.2011; 1 o', 1 q [9], p.t., 7-17.07.2010; 1 + [9], p.t., 17-27.07.2010.

Pardosa prativaga (L. Koch, 1870)* - 1 ○ [6], p.t., 11-21.06.2011.

Pirata piraticus (Clerck, 1758)* -1 \% [5], p.t., $7-$ 17.07.2010; 1 ○, 2 ㅇ [6], p.t., 7-17.07.2010; 2 ㅇ [6], p.t., 11-21.06.2011; $2 \bigcirc^{7} \sigma^{7}, 2$ OO [6], p.t., 21.061.07.2011; 1 ○, 3 ㅇ [6], p.t., 1-11.07.2011; 2 우 [6], p.t., 21-31.07.2011.

Pirata piscatorius (Clerck, 1758)* - $1 \bigcirc^{7}[1]$, p.t., 11-21.06.2011; 2 o $^{7}$ [6], p.t., 11-21.06.2011.

Piratula hygrophilus (Thorell, 1872)* - 3 +o [4], p.t., 7-17.07.2010; $5 \sigma^{7} \sigma^{7}, 1$ O, 1 juv. [4], p.t., 11-

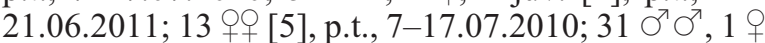
[5], p.t., 11-21.06.2011; $1 \sigma^{\text {T }}$ [5], p.t., 1-11.07.2011; 1, 5 juv. [5], bc., 26.06.2011; 1 क, 21 juv. [5], bc., 6.07.2011; 1 , juv. [5], bc., 16.07.2011; 8 o+ [6], p.t., 7-17.07.2010; 74 O $^{7}, 1 \sigma^{7}$ sbad, 6 oᄋ [6], p.t., 1121.06.2011; $24 \sigma^{7} \sigma^{\top}, 26$ o+, 2 juv. [6], p.t., 21.06$1.07 .2011 ; 5 \bigcirc^{7} \sigma^{7}, 5$ 우 [6], p.t., 1-11.07.2011; 3 우, 1 cocoon [6], p.t., 11-21.07.2011; 8 우 [6], p.t., 21$31.07 .2011 ; 1$ क with cocoon [6], bc., 27.06.2011; 2 ㅇ [6], bc., 27.06.2011; 2 +o with cocoons [6], bc., 7.07.2011; 1 O $^{7}$ [7], p.t., 11-21.06.2011.

Tarentula accentuata (Latreille, 1817)* - 2 오 [2], p.t., 11-21.06.2011; 1 \% [8], p.t., 11-21.06.2011; 1 + [8], p.t., 21.06-1.07.2011.

Tarentula aculeata (Clerck, 1758)* - $1 \sigma^{\top}[1]$, p.t., 21.06-1.07.2011; 1 O $^{\top}, 1$ + [1], p.t., 21.06$1.07 .2011 ; 1$ [3], p.t., 7-17.07.2010; $3 \sigma^{7} \sigma^{7}[3]$, p.t.,
11-21.06.2011; $2 \sigma^{7} \sigma^{7}[3]$, p.t., 21.06-1.07.2011; $1 \sigma^{7}$ [4], p.t., 11-21.06.2011; $2 \sigma^{7} \sigma^{7}$ [9], p.t., 11-21.06.2011.

Tarentula cuneata (Clerck, 1758)* - 4 90 [1], p.t., 11-21.06.2011; 5 우 [1], p.t., 21.06-1.07.2011; 3 우 [1], p.t., $1-11.07 .2011 ; 4$ 우 [2], p.t., 717.07.2010; 1 ㅇ [2], p.t., 17-27.07.2010; 10 우 [2], p.t., 11-21.06.2011; 2 +中, 1 juv. [2], p.t., 1-11.07.2011; 2 +o [9], p.t., 1-11.07.2011.

Tarentula fabrilis (Clerck, 1758)* -5 0 [ [2], p.t., 11-21.06.2011; 1 ㅇ [2], p.t., 21.06-1.07.2011; 2 우 [8], p.t., 11-21.06.2011.

Tarentula inquilina (Clerck, 1758)* - 1 ㅇ [1], p.t., 11-21.06.2011; 1 \% [5], p.t., 21.06-1.07.2011.

(-) Tarentula schmidti (Hahn, 1835). MR: wheat fields [Timraleev, 1998].

Tarentula sulzeri Pavesi, 1873* - $10 \sigma^{7} \sigma^{7}[1]$, p.t., 11-21.06.2011; $6 \sigma^{\top} \sigma^{\top}$ [1], p.t., 21.06-1.07.2011; $1 \sigma^{7}$ [1], p.t., 1-11.07.2011; 2 ठ $\sigma^{\top}$ [2], p.t., 1121.06.2011; $3 \sigma^{7} \sigma^{7}$ [3], p.t., 21.06-1.07.2011.

Trochosa ruricola (De Geer, 1778) - $10 \bigcirc^{7} \sigma^{7}, 13$ 우 [1], p.t., 11-21.06.2011; $2 \bigcirc^{\top} \sigma^{\top}, 16$ 우 [1], p.t., 21.06-1.07.2011; 3 우 [1], p.t., 1-11.07.2011; 1 ㅇ [2], p.t., 21-31.07.2011; 1 ๙ , 1 \%, 7 juv. [3], p.t., 1121.07.2011; 1 + [3], bc. 3, 24.06.2011; 1 \%, 3 juv. [4], 21.06-1.07.2011; 2 ठ $\sigma^{7}$ [4], p.t., 21.06-1.07.2011; 2 우, 2 juv. [4], 21-31.07.2011; 1 [7], p.t., 1121.07.2011; 1 \% [9], p.t., 1-11.07.2011. MR: wheat fields [Timraleev, 1998].

Trochosa spinipalpis (F.O. Pickard-Cambridge, $1895)^{*}-1 \sigma^{7}$ [6], p.t., 1-11.07.2011.

(-) Trochosa terricola Thorell, 1856. MR: wheat fields [Timraleev, 1998].

Xerolycosa miniata (C.L. Koch, 1834) - $1 \sigma^{7}, 4$ 우 [1], p.t., 7-17.07.2010; $8 \sigma^{7} \sigma^{7}, 1$ juv. [1], p.t., 21.061.07.2011; 4 О $^{7}, 12$ O9, 1 juv. [2], p.t., 7-17.07.2010; $25 \sigma^{7} \sigma^{7}, 5 \sigma^{7} \sigma^{7}$ sbad, 45 o+, 7 juv. [2], p.t., 1727.07.2010; $21 \sigma^{\top} \sigma^{\top}, 7$ o+, 3 juv. [2], p.t., $11-$ 21.06.2011; 7 ○ $^{7}$ [2], p.t., 21.06-1.07.2011; $18 \sigma^{7} \sigma^{7}$, 5 우 [2], p.t., 1-11.07.2011; $2 \sigma^{\top} \sigma^{\top}, 2$ 우 [2], p.t., 2131.07.2011; 7 O $^{7}, 2$ 우 [8], p.t., 11-21.06.2011; 4 O $^{7} \sigma^{7}$ [8], p.t., 21.06-1.07.2011; $1 \sigma^{7}$ [8], p.t., 1-11.07.2011; 1 [9], p.t., 7-17.07.2010; 2 ○ $\sigma^{7}$ [9], p.t., 1-11.07. 2011; 2 O' $\sigma^{7}, 5$ + [10], p.t., 7-17.07.2010. MR: wheat fields [Timraleev, 1998].

\section{PHILODROMIDAE (4)}

Philodromus cespitum (Walckenaer, 1802)* - 1

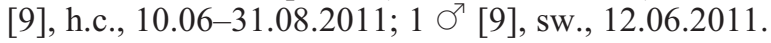
Philodromus fuscomarginatus (De Geer, 1778)* 1 [3], h.c., 10.06-31.08.2011.

Thanatus arenarius Thorell, 1872* -1 ○ [2], p.t., 11-21.06.2011; $1 \sigma^{\top}$ [8], p.t., 11-21.06.2011.

(-) Tibellus maritimus (Menge, 1875). MR: wheat fields [Timraleev, 1998].

Tibellus oblongus (Walckenaer, 1802) - 1 \% [2], sw., 17-27.07.2010; 1 q [2], p.t., 17-27.07.2010; 1 \%

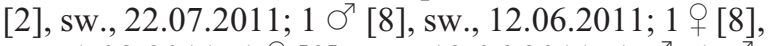
Sw., 1.08.2011; 1 क [9], sw., 12.06.2011; 1 O $^{7}, 1$ O', 1 juv. [9], sw., 22.06.2011. MR: wheat fields [Timraleev, 1998]. 


\section{PHOLCIDAE}

(-) Pholcus phalangioides (Fuesslin, 1775). MR: Ardatovo Distr.: Smol'kovo Vil. [Budilov, Timoshkina, 2010].

PISAURIDAE (2)

Dolomedes fimbriatus (Clerck, 1758)* - 1 ○ [1], p.t., 1-11.07.2011.

Pisaura mirabilis (Clerck, 1758) - 1 juv. [1], p.t., 7-17.07.2010; 1 juv. [6], p.t., 7-17.07.2010. MR: wheat fields [Timraleev, 1998].

SALTICIDAE (10)

Ballus depressus (Walckenaer, 1802)* - $1 \overbrace{}^{7}$, 2.08.1936.

Dendryphantes rudis (Sundevall, 1832)* - 1 ठ [7], sw., 27.07-6.08.2010.

Evarcha arcuata (Clerck, 1758)* - 2 우 [1], sw., 17-27.07.2010; 1 + 2 juv. [1], sw., 22.06.2011; 1 ,

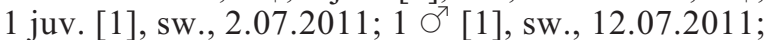
$1 \bigcirc^{7}$ [1], sw., 22.07.2011; 1 [ [1], sw., 1.08.2011; 1 क

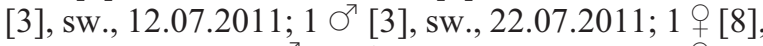
sw., 11.08.2011; 1 ○ [9], h.c., 17-27.07.2010; 1 \& [9], h.c., 10.06-31.08.2011; 1 ठ [9], sw., 21.08.2011.

Evarcha falcata (Clerck, 1758) - $1 \sigma^{7}$ [3], sw., 27.07-6.08.2010; 1 q [3], bc., 14.06.2011; 1 9, 3 juv. [3], sw., 12.07.2011; 2 우 [3], sw., 12.07.2011; 1 ठ [7], sw., 11.08.2011; 1 क [9], sw., 17-27.07.2010. MR: wheat fields [Timraleev, 1998].

Heliophanus cupreus (Walckenaer, 1802) -1 o [9], sw., 2.07.2011. MR: wheat fields [Timraleev, 1998].

Heliophanus dubius C.L. Koch, 1835* - 1 \% [3], sw., 7-17.07.2010.

Heliophanus flavipes (Hahn, 1832) - 3 우, 2 juv. [2], sw., 7-17.07.2010; 1 \%, 6 juv. [2], sw., 27.07$6.08 .2010 ; 1 \sigma^{\top}[2]$, p.t., $1-11.07 .2011 ; 1$ \% [8], sw.,

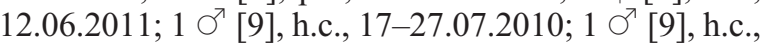
10.06-31.08.2011. MR: wheat fields [Timraleev, 1998].

Phlegra fasciata (Hahn, 1826)* — 2 우 [9], p.t., 17-27.07.2010.

Pseudeuophrys obsoleta (Simon, 1868)* - $10^{7}$ [3], p.t., 7-17.07.2010.

Sitticus saltator (Simon, 1868)* - 1 + [2], sw., 717.07.2010.

\section{TETRAGNATHIDAE (8)}

Metellina segmentata (Clerck, 1758)* - 1 \% [6],

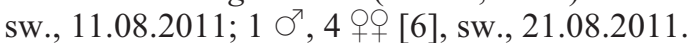

Pachygnatha clercki Sundevall, 1823* - $1 \sigma^{7}[6]$, sw., 21.08.2011.

Pachygnatha degeeri Sundevall, $1830-2$ 우 [5], bc., 27.07-6.08.2010; 1 ठ [9], p.t., 7-17.07.2010. MR: wheat fields [Timraleev, 1998].

Pachygnatha listeri Sundevall, 1830* - 1 ○', 2 우 [5], bc., 17-27.07.2010; 1 \% [5], p.t., 11-21.06.2011;

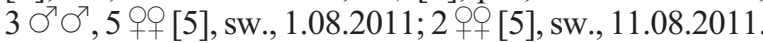

Tetragnatha dearmata Thorell, 1873* - $1 \sigma^{7}$ [5],

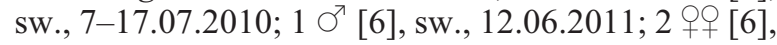
sw., 22.06.2011.

Tetragnatha extensa (L., 1758) - 1 ア , 1 q [1], sw., 22.06.2011. MR: wheat fields [Timraleev, 1998].
Tetragnatha obtusa C.L. Koch, $1837^{*}-1 \bigcirc^{7}$ [9], h.c., 10.06-31.08.2011.

Zygiella stroemi (Thorell, 1875)* — 1 \% [9], h.c., 10.06-31.08.2011.

THERIDIIDAE (12)

Crustulina guttata (Wider, 1834)* $-1 \sigma^{\top}$ [3], bc., 14.07.2011.

Enoplognatha ovata (Clerck, 1758)* - 2 juv. [3], sw., 12.06.2011; 1 O $^{7}$ sbad. [3], sw., 22.06.2011; 2 O $^{7} \sigma^{7}$ [3], sw., 2.07.2011; 3 oq, 1 juv. [3], sw., 2.07.2011; 1 juv. [5], sw., 12.06.2011; 1 [6], sw., 12.06.2011; 1 juv. [6], sw., 22.06.2011; 1 q [6], sw., 12.07.2011; 1 + [7], sw., 12.07.2011.

Euryopis flavomaculata (C.L. Koch, 1836)* - $10^{7}$ [5], p.t., 21.06-1.07.2011.

Neottiura bimaculata (L., 1767)* $-1 \bigcirc^{7}$ [3], sw., 2.07.2011.

Phylloneta impressa (L. Koch, 1881)* - 1 \% [2], p.t., 7-17.07.2010; 1 [ [4], h.c., 17-27.07.2010; 1 ठ [8], sw., 12.06.2011.

Platnickina tincta (Walckenaer, 1802)* -1 \& [4], sw., 7-17.07.2010.

Robertus arundineti (O. Pickard-Cambridge, 1871)* -2 우 [5], bc., 7-17.07.2010; $2 \bigcirc^{7} \sigma^{7}$ [5], bc., 17-27.07.2010; 1 疋 [5], p.t., 21.06-1.07.2011; 1 ऊ [6], bc., 7-17.07.2010; 1 + [9], bc., 17-27.07.2010.

Robertus lividus (Blackwall, 1836)* - $10^{7}$ [4], bc., 15.06.2011; 1 q [6], bc., 26.08.2011.

Steatoda albomaculata (De Geer, 1778) - $1 \sigma^{7}$ [8], p.t., 11-21.06.2011; 1 ठ [8], p.t., 21-31.07.2011. MR: wheat fields [Timraleev, 1998].

Steatoda castanea (Clerck, 1758)* $-1+$ [9], h.c., summer 2011.

Steatoda phalerata (Panzer, 1801)* -1 q [2], p.t., 7-17.07.2010; 1 \%, 1 juv. [2], p.t., 17-27.07.2010; 3 ○ $\sigma^{7}$ [2], p.t., 11-21.06.2011; 1 ㅇ [3], p.t., 21.061.07.2011; $1 \bigcirc^{\top}, 2$ 우 [8], p.t., 11-21.06.2011.

Theridion varians (Hahn, 1833)* - 1 [4], sw., 7-17.07.2010; 1 O $^{7}[6]$, sw., 12.06.2011; 1 \% [7], sw., 7-17.07.2010; 1 ㅇ [8], sw., 17-27.07.2010.

\section{TITANOECIDAE}

(-) Titanoeca schineri L. Koch, 1872. MR: wheat fields [Timraleev, 1998].

\section{THOMISIDAE (12)}

Coriarachne depressa (C.L. Koch, 1837)* - $1 \bigcirc^{7}$, 18.08.1936.

(-) Ebrechtella tricuspidata (Fabricius, 1775). MR: wheat fields [Timraleev, 1998].

(-) Heriaeus oblongus Simon, 1918. MR: wheat fields [Timraleev, 1998].

Misumena vatia (Clerck, 1758) - 1 \%, 2 juv. [1], sw., 12.07.2011; 1 [9], h.c., 17-27.07.2010; 1 o $^{7}$ [9], sw., 12.06.2011. MR: Ardatovo Distr.: Smol'kovo Vil. [Budilov, Timoshkina, 2010].

Ozyptila praticola (C.L. Koch, 1837) - $1 \sigma^{7}$ [4], p.t., 11-21.06.2011; $4 \sigma^{\top} \sigma^{\top}$ [4], p.t., 21.06-1.07.2011; $1 \sigma^{7}$ [4], p.t., 1-11.07.2011; $1 \sigma^{7}$ [4], bc., 4.08.2011; $1 \sigma^{7}$ [6], p.t., 21.06-1.07.2011; 1 [6], bc., 7.07.2011; 1 
[6], bc., 6.08.2011; 1 q [8], p.t., 27.07-6.08.2010; 1 ㅇ [9], bc., 30.07.2011; 1 \% [9], bc., 29.08.2011. MR: Ardatovo Distr.: Smol'kovo Vil. [Budilov, Timoshkina, 2010]. 2010.

Ozyptila pullata (Thorell, 1875)* -1 + , no label,

Ozyptila trux (Blackwall, 1846)* -1 [5], p.t., 21.06-1.07.2011.

Xysticus audax (Schrank, 1803)* - 1 ○' [2], p.t., 21.06-1.07.2011; 2 oq [3], p.t., 7-17.07.2010; 1 ○ [3], p.t., 21.06-1.07.2011.

Xysticus cristatus (Clerck, 1758)* -1 [7], p.t., 7-17.07.2010.

Xysticus kempeleni Thorell, 1872* $-1+$ [3], p.t., 11-21.06.2011.

Xysticus kochi Thorell, $1872-1$ \& [2], p.t., 21.061.07.2011. MR: wheat fields [Timraleev, 1998].

Xysticus luctator L. Koch, 1870* - 19 [3], p.t., 7

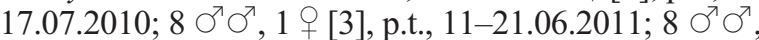
3 of, 1 juv. [3], p.t., 21.06-1.07.2011; $1 \sigma^{7}$ [3], p.t., 11-21.07.2011; 4 O O $^{7}$ [4], p.t., 21.06-1.07.2011; 4 $\sigma^{7} \sigma^{7}[7]$, p.t., $11-21.06 .2011$.

Xysticus luctuosus (Blackwall, 1836)* - $3 \sigma^{7} \sigma^{7}$ [3], p.t., 11-21.06.2011; 2 ○ $\sigma^{\top}$ [3], p.t., 21.061.07.2011; 1 \% [3], h.c., 10.06-31.08.2011; 1 ○ [4], p.t., 21.06-1.07.2011; $1 \sigma^{7}$ [7], p.t., 11-21.06.2011.

Xysticus striatipes L. Koch, $1870-1 \sigma^{7}$ [8], sw., 11.08.2011. MR: wheat fields [Timraleev, 1998].

Xysticus ulmi (Hahn, 1831)* — 2 우 [1], sw., 22.06.2011; 2 우 [1], sw., 12.07.2011; 1 [9], h.c., 10.06-31.08.2011.

\section{ZORIDAE (2)}

Zora nemoralis (Blackwall, 1861)* -1 \% [7], p.t., 17-27.07.2010; 1 ○ [7], p.t., 11-21.06.2011.

Zora spinimana (Sundevall, 1832)* $-1+$ [9], bc., 30.07.2011.

In two field seasons (2010 and 2011), a total of 142 spider species of 17 families has been recorded from the MSR; of them 121 species are new to the MR fauna. The entire MSR spider species number seems to include as many as 250-300 species. To date, 161 spider species of 19 families have been recorded from Mordovian Republic. The species diversity of eight main spider families in the MSR is close to that of Russain Plain (cf. Mikhailov [2013, fig. 2]). The respective shares are as follows: Linyphiidae - $26 \%$ in the MSR vs. 30\% in Russan Plain, Lycosidae - $13 v s$. $9 \%$, Gnaphosidae - 10 vs. $13 \%$, Theridiidae - $8 v s$. $7 \%$, Thomisidae - $8 v$ s. 5\%, Salticidae - $7 v s .10 \%$, Araneidae -6 vs. 5\%, Tetragnathidae -6 vs. less than $3 \%$. We have no generalized comparable data on the spider species diversity of mixed forest and foreststeppe zones of Russian Plain, but the closest comparative data are available for the Zvenigorod Biological Station of the Moscow Lomonosov State University situated in the mixed forest zone [Mikhailov, 1983; one season, biocenometer and hand collecting, 130 species], as well as both for the 'Privolzhskaya Leso- step' [Polchaninova, 2008, one season, various collecting methods, 128 species] and 'Tsentralno-Chernozyomnyi' [Polchaninova, 2009, three seasons, various collecting methods, 167 species] reserves situated in the forest-steppe zone. A comparative decrease of the linyphiid share from the forest $(43.1 \%$ in Zvenigorod Biological Station) to forest-steppe (22\% in 'Privolzhskaya Lesostep' and 30.5\% in 'Tsentralno-Chernozyomnyi' Reserve) zones has been observed. The MSR data ( $25 \%$ of linyphiids) is closer to those from the forest-steppe than to that of the forest zone. However, such result seems to be an aberration of the sampling methods used: the linyphiids are more numerous in biocenometer samples than in pitfall traps and sweeping (K. Mikhailov, pers. data). To summarize this brief discussion, both the MSR and MR spider faunas merit further detailed investigations.

ACKNOWLEDGEMENTS. We are very grateful to Dr A.V. Ruchin, Director of the Mordovian State Reserve, for his support of the second author in the 2010-2011 field seasons, as well as to Dr A.V. Tanasevitch (Moscow, Russia) for help in the identification of Linyphiidae. Dr M.K. Ryzhov (Saransk, Russia) kindly supplied us with rare papers on spiders of Mordovia. Dr D.V. Logunov (Manchester, UK) is deeply obliged for both logistic and linguistic help.

\section{References}

Budilov V.V., Timoshkina O.I. 2010. [Some aspects of the study of spiders of Mordovian Republic] // Aktual'nye problemy zoologii, ekologii, metodiki obucheniya i pedagogiki (po materialam zasedaniya sektsii “Aktual'nye problemy zoologii, ekologii, metodiki obucheniya biologii v obshcheobrazovatel'noi shkole" v ramkakh Vserossiyskoi nauchno-prakticheskoi konferentsii “46 Evsevievskie chteniya” (19-20 maya 2010 g.)). Saransk. P.13-15 [in Russian].

Mikhailov K.G. 1983. [Spiders (Arachnida, Aranei) of forest litter of Zvenigorod Biological Station, MSU] // M.S. Ghilarov (ed.). Fauna i ekologiya pochvennykh bespozvonochnykh Moskovskoi oblasti. Moscow: Nauka Publ. P.52-67 [in Russian].

Mikhailov K.G. 2013. Advances in the study of the spider (Aranei) fauna of Russia and adjacent regions: a 2011 update // Arthropoda Selecta. Vol.22. No.1. P.47-53.

Polchaninova N.Yu. 2008. [Materials to the spider fauna (Araneae) of the Ostrovtsovsky part of the 'Privolzhskaya lesostep' $\mathrm{Na}$ ture reserve (Penza Area, Russia)] // Caucasian Entomol. Bull. Vol.4. No.2. P.155-161 [in Russian, with English summary].

Polchaninova N.Yu. 2009. [Spiders (Araneae) of the Streletsky part if the Tsentralno-Chernozwmny Nature reserve (Kursk region)] // Caucasian Entomol. Bull. Vol.5. No.1. P.13-27 [in Russian, with English summary].

Redikortsev V. 1938. [Materials to the entomofauna of Mordovian State reserve] // Fauna Mordovskogo gosudarstvennogo zapovednika im. P.G. Smidovicha. Moscow: Komitet po zapovednikam pri Prezidiume VTsIK. P.137-146 [in Russian].

Timraleev Z.A. 1998. [Fauna and ecology of spiders of wheat fields of Mordovia] // Vodnye i nazemnye ekosistemy i okhrana prirody Levoberezhnogo Prisurya. Saransk. P.82-86 [in Russian]

Fauna Mordovskogo gosudarstvennogo zapovednika im. P.G. Smidovicha [Proceedings of the P.G. Smidovich Mordovian State Reserve]. 1938. Moscow: Komitet po zapovednikam pri Prezidiume VTsIK. 154 p. [in Russian] 